\title{
Elastic-Plastic Vibration of a Rod
}

\author{
By \\ Tetsuhiko Mryoshi*
}

\section{Introduction}

The following equation is a mathematical model to represent the elasticplastic vibration of a straight uniform rod submitted to longitudinal impact.

$$
\dot{\sigma}= \begin{cases}k \dot{u}_{x}-\sigma_{x}=0 \\ (1-\xi) k \dot{u}_{x} & \text { in plastic region }\end{cases}
$$

where $\xi, k$ are positive constants and $0<\xi<1$ ([2]).

In this paper we prove that there is a unique weak solution to the initialboundary value problem of this equation and it is obtained as a limit of the finite element solutions.

This kind of problems is treated by Duvaut-Lions [1]. They define an initial value problem involving an inequality as a weak form of the original problem and have obtained a solution by a penalization technique. However, the relation between the original problem and their problem is not necessarily clear in their formulation and, as the result, numerical method to solve the problem is restricted within a certain class. The penalization technique is used by Johnson [3] to get existence theorems in static problems with hardening.

We also derive a weak form including an inequality. However, the basic idea to get the solution is not the penalization but a discretization, so that the relation between the original problem and the derived one is quite clear.

We thus start from the vibration of a single masspoint system, then proceed to a multiple masspoint system and to the continuous case.

\section{§1. Elastic-Plastic Vibration of a Single Masspoint System}

1.1. Equation of motion. Let us consider the vibration of a single

Communicated by S. Hitotumatu, July 1, 1978.

* Department of Mathematics, Kumamoto University. 
masspoint system described by the following initial value problem.

$$
\rho \ddot{u}+\sigma=0 \text { in } T \text {, }
$$

where $\rho$ : positive constant, $T=(0, T), u(0)=0, \dot{u}(0)$ : given, and the yield displacement $\bar{u}^{(0)}(>0)$ is given.

The function $\sigma$ is a continuous function and satisfies:

$$
\begin{array}{ll}
\dot{\sigma}=k \dot{u} & \text { if the system is elastic, } \\
\dot{\sigma}=(1-\xi) k \dot{u} & \text { if the system is plastic. }
\end{array}
$$

We define the states "elastic" and "plastic" as follows. First, put $\sigma=k u$. Assume that the solution of the equation (1.1) satisfies $|u(t)|=\bar{u}^{(0)}$ at $t=t_{0}$ for the first time. Then we define that the system is elastic for the time interval $\left[0, t_{0}\right)$. (Note that the definition of "elastic" is not independent of the solution of the initial value problem. These are determined at the same time.) If $\dot{u}\left(t_{0}\right) \neq 0$, then the system is defined to be plastic for $t \geq t_{0}$. If $\dot{u}\left(t_{0}\right)=0$, the state for $t \geq t_{0}$ is determined by the following check. (ABC)-check:

(A) If $\dot{u}$ converges to 0 from above:

(1) if $\ddot{u}\left(t_{0}\right) \geq 0$ then plastic for $t \geq t_{0}$,

(2) if $\ddot{u}\left(t_{0}\right)<0$ then elastic for $t \geq t_{0}$ (case (A)).

(B) If $\dot{u}$ converges to 0 from below:

(1) if $\ddot{u}\left(t_{0}\right)>0$ then elastic for $t \geq t_{0}$ (case (B)),

(2) if $\ddot{u}\left(t_{0}\right) \leq 0$ then plastic for $t \geq t_{0}$.

(C) If $\dot{u}$ converges to 0 vibrating. Plastic for $t \geq t_{0}$.

Remark. If $\dot{u}(t)=\ddot{u}(t)=0$ at $t=t_{0}$ then all $\stackrel{(k)}{u}\left(=\frac{d^{k} u}{d t^{k}}\right)(k \geq 3)$ vanish at $t=t_{0}+0$ independently of the state for $t \geq t_{0}$, so that $u=u\left(t_{0}\right), \sigma=0$ is the only possible solution for $t \geq t_{0}$. Observe that this (ABC)-check is a formal classification for logical consistency.

Subsequent state of the system is determined recursively as follows.

(I) The case when the present state is plastic. Assume that the present state began at $t=t_{m}$, and the solution of the equation (1.1) satisfies $\dot{u}(t)=0$ at $t=t_{m+1}$ $\left(>t_{m}\right)$ for the first time. Then we define the system is plastic for the time interval $\left[t_{m}, t_{m+1}\right)$. The state for $t \geq t_{m+1}$ is determined by the (ABC)-check.

(II) The case when the present state is elastic. Assume that the present state began at $t=t_{m}$ after the (ABC)-check.

(1) Case $(A)$. Assume that the solution $u$ satisfies 


$$
u(t) \leq u\left(t_{m}\right)-2 \bar{u}^{(0)},
$$

or

$$
u(t)=u\left(t_{m}\right)
$$

at $t=t_{m+1}\left(>t_{m}\right)$ for the first time. Then we define the system is elastic for the time interval $\left[t_{m}, t_{m+1}\right)$.

(2) Case (B). Assume that the solution $u$ satisfies

$$
u(t) \geq u\left(t_{m}\right)+2 \bar{u}^{(0)},
$$

or

$$
u(t)=u\left(t_{m}\right)
$$

at $t=t_{m+1}\left(>t_{m}\right)$ for the first time. Then we define the system is elastic for the time interval $\left[t_{m}, t_{m+1}\right)$.

The state for $t \geq t_{m+1}$ is determined as

(II) $a$ if $\dot{u} \neq 0$, the system is plastic for $t \geq t_{m+1}$,

(II) $b$ if $\dot{u}=0$, the state for $t \geq t_{m+1}$ is determined by the (ABC)-check.

Our initial value problem is well posed by the above procedure and has a unique $C^{2}$-class solution. The hardening in the above rule corresponds to the kinematic hardening.

1.2. Energy of the single masspoint system. Let $u^{(j)}(j=0,1,2, \ldots)$ be the displacement at which the $(j+1)$-th change of the state occurs. We say that the system is at stage $(m)$ if the change of the state occurred $m+1$ times in the past.

The key to derive an energy equality for the present problem and also to develop our theory in this paper is the following simple theorem which represents the initial value problem by a single equation.

Theorem 1.1. The equation (1.1) is represented as follows, if the system is at stage $(m)$.

$$
\rho \ddot{u}+k u-\xi k \sum_{0}^{m}(-1)^{j}\left(u-u^{(j)}\right)=0 .
$$

Proof. Induction on $m$. The equation holds for $m=0$. Assume that (1.2) holds until $m=r(\geq 0)$.

(1) If stage $(r)$ is elastic: Since stage $(r+1)$ is plastic, $\dot{\sigma}$ is given by $(1.1)_{b}$. Let $t_{r+1}$ be the time at which stage $(r+1)$ starts. Integrating $(1.1)_{b}$, we have

$$
\sigma(t)=\sigma\left(t_{r+1}\right)+(1-\xi) k\left[u-u^{(r+1)}\right] .
$$

On the other hand, by the assumption of the induction, the following equality holds at stage( $r$. 


$$
\sigma(t)=k u-\xi k \sum_{0}^{r}(-1)^{j}\left(u-u^{(j)}\right) .
$$

Therefore, by the continuity of $\sigma$, it holds at $t=t_{r+1}$

$$
\begin{aligned}
\sigma\left(t_{r+1}\right) & =k u^{(r+1)}-\xi k \sum_{0}^{r}(-1)^{j}\left(u^{(r+1)}-u^{(j)}\right) \\
& =k u^{(r+1)}+\xi k \sum_{0}^{r}(-1)^{j} u^{(j)} \quad(r: \text { odd }) .
\end{aligned}
$$

Substituting this into (1.3), we have

$$
\begin{aligned}
\sigma(t) & =k u^{(r+1)}+\xi k \sum_{0}^{r}(-1)^{j} u^{(j)}+(1-\xi) k\left(u-u^{(r+1)}\right) \\
& =(1-\xi) k u+\xi k \sum_{0}^{r+1}(-1)^{j} u^{(j)} \\
& =k u-\xi k \sum_{0}^{r+1}(-1)^{j}\left(u-u^{(j)}\right) .
\end{aligned}
$$

Therefore (1.2) holds for $m=r+1$.

(2) If stage $(r)$ is plastic: The situation is completely the same as above.

From this equation we can easily derive a simple energy equality which represents the non-conservation of energy.

Theorem 1.2. Let $E_{m}$ be defined by

$$
E_{m}(t)=\frac{\rho}{2}(\dot{u})^{2}+\frac{k}{2} u^{2}-\frac{\xi k}{2} \sum_{0}^{m}(-1)^{j}\left(u-u^{(j)}\right)^{2} .
$$

Then the following equality holds at stage $(m)$.

$$
E_{m}(t)=E_{0}
$$

where $E_{0}$ is the initial energy.

Proof. Let $t_{m}$ be the time at which the stage $(m)$ starts. Multiplying by $\dot{u}$ to (1.2) and integrating from $t_{m}$ to $t$, we have

$$
\begin{aligned}
E_{m}(t) & =\frac{\rho}{2}(\dot{u})^{2}\left(t_{m}\right)+\frac{k}{2}\left(u^{(m)}\right)^{2}-\frac{\xi k}{2} \sum_{j=0}^{m-1}(-1)^{j}\left(u^{(m)}-u^{(j)}\right)^{2} \\
& =E_{m-1}\left(t_{m}\right) .
\end{aligned}
$$

This implies that the quantity defined by (1.4) is constant through all stages. Therefore it is equal to the initial energy.

Remark. This theorem implies that the elastic-plastic vibration converges to an elastic vibration as $t \rightarrow \infty$. This is the case also for the multiple masspoint 
system considered later.

1.3. A weak form of the equation of motion. The initial value problem formulated above can be represented simply as follows.

Theorem 1.3. The initial value problem of the single masspoint system is equivalent to the following problem: Define

$$
K=K_{\alpha}=\left\{\tau \in C(T) ;|\tau-\alpha| \leq \sigma_{0} \text { for any } t \in T\right\} ; \sigma_{0}=k \bar{u}^{(0)} .
$$

Seek $v, \sigma, \alpha$ which are differentiable and satisfy, for all $t \in T$

$$
\left\{\begin{array}{l}
(\dot{\sigma}-k v, \tau-\sigma) \geq 0 \quad \text { for all } \quad \tau \in K \\
\dot{\alpha}=\left(1-\frac{1}{\xi}\right)(\dot{\sigma}-k v) \\
\rho \dot{v}+\sigma=0
\end{array}\right.
$$

and $\sigma \in K, \sigma(0)=0, \alpha(0)=0, v(0)=\dot{u}(0)$.

Remark. $(x, y)=x y$ in this case. This is for the generalization of our method to more complicated problems.

Proof of the Theorem. Let $v=\dot{u}$ and $\alpha$ be the parameter representing the movement of the center of the yield surface (two points, in this case). We then show that the solution $u, \sigma$ of the equation (1.1) satisfy (1.5). If the system is elastic, then $\dot{\sigma}-k v=0, \dot{\alpha}=0$ and thus (1.5) holds well. If the system is plastic, then $\dot{\sigma}=(1-\xi) k v$, so that $(\dot{\sigma}-k v, \tau-\sigma)=-\xi k(v, \tau-\sigma)$. In this case,

$$
\begin{aligned}
& \text { if } v=\dot{u}>0, \text { then } \sigma=\alpha+\sigma_{0}, \\
& \text { if } v=\dot{u}<0, \text { then } \sigma=\alpha-\sigma_{0} .
\end{aligned}
$$

Therefore, in any cases we have

$$
-\xi k(v, \tau-\sigma) \geq 0 \quad \text { for any } \tau \in K \text {. }
$$

Also, $\dot{\alpha}=\left(1-\frac{1}{\xi}\right)(-\xi k v)=(1-\xi) k v=\dot{\sigma}$, which is certainly the equation for $\alpha$. Therefore $u$ and $\sigma$ satisfy (1.5).

The proof is complete if we can show the uniqueness of the solution for (1.5). Substitute the second equation into the first inequality. We then have

$$
(\dot{\alpha}, \tau-\sigma) \leq 0 \quad \text { for any } \quad \tau \in K .
$$

Let $\theta$ be an arbitrary continuous function satisfying $|\theta| \leq 1$. Then the function of the form

$$
\tau=\alpha+\sigma_{0} \theta
$$


is included in $K$. Therefore we have

$$
\left(\dot{\alpha}, \alpha+\sigma_{0} \theta-\sigma\right) \leq 0 \quad \text { for any such } \theta .
$$

Assume that there is another solution $\left(v_{*}, \sigma_{*}, \alpha_{*}\right)$. Obviously

$$
\left(\dot{\alpha}_{*}, \alpha_{*}+\sigma_{0} \theta-\sigma_{*}\right) \leq 0 \quad \text { for any such } \theta .
$$

Put $\theta=\left(\sigma_{*}-\alpha_{*}\right) / \sigma_{0}$ in (1.6) and $\theta=(\sigma-\alpha) / \sigma_{0}$ in (1.7), and add the both inequalities. Then we have

$$
\left(\dot{\alpha}-\dot{\alpha}_{*}, \alpha-\alpha_{*}-\left[\sigma-\sigma_{*}\right]\right) \leq 0 .
$$

By using two equations of (1.5), we have

$$
\left(\dot{\alpha}-\dot{\alpha}_{*}, \sigma-\sigma_{*}\right)=\left(1-\frac{1}{\zeta}\right)\left[\frac{1}{2}\left\|\sigma-\sigma_{*}\right\|_{t}^{2}+\rho \frac{k}{2}\left\|v-v_{*}\right\|_{t}^{2}\right] .
$$

Hence we have

$$
\left\|\alpha-\alpha_{*}\right\|^{2}-\left(1-\frac{1}{\xi}\right)\left[\left\|\sigma-\sigma_{*}\right\|^{2}+\rho k\left\|v-v_{*}\right\|^{2}\right] \leq 0,
$$

which implies $\alpha=\alpha_{*}, \sigma=\sigma_{*}$ and $v=v_{*}$. This completes the proof.

1.4. Energy inequality. A basic energy inequality is derived from (1.5). We see the inequality in (1.5) implies

$$
(\dot{\sigma}, \sigma)+k(\dot{v}, v)-(\dot{\sigma}-k v, \tau) \leq 0 .
$$

Here we can put $\tau=\alpha$. Therefore we have

$$
(\dot{\sigma}, \sigma)+k(\dot{v}, v)+\frac{\xi}{1-\xi}(\dot{\alpha}, \alpha) \leq 0,
$$

which implies

$$
\frac{\xi}{1-\xi}(\alpha, \alpha)+(\sigma, \sigma)+k(v, v) \leq k(v, v)(0)
$$

\section{§2. Elastic-Plastic Vibration of a Multiple Masspoint System}

2.1. Equation of motion. All results obtained in the preceding section are extended formally to the multiple system.

Let $u_{i}(i=0,1, \ldots, N)$ be the displacement of the $i$-th masspoint (we assume $\left.u_{0}=0\right)$. Let $\rho_{i}, k_{i}$ and $\xi_{i}$ be the mass, stiffness and plasticity factor of the $i$-th masspoint. We introduce the quantity $U_{i}=u_{i}-u_{i-1}$ which corresponds to the strain at $i$-th point. Then the equation of motion of this system is written as 


$$
\rho_{i} \ddot{u}_{i}+\sigma_{i}\left(U_{i}\right)-\sigma_{i+1}\left(U_{i+1}\right)=0 \quad i=1,2, \ldots, N,
$$

where $\sigma_{i}\left(U_{i}\right)(i=1,2, \ldots, N+1)$ is a continuous function of $t$ such that

$$
\begin{array}{ll}
\dot{\sigma}_{i}\left(U_{i}\right)=k_{i} \dot{U}_{i} & \text { if the } i \text {-th point is elastic, } \\
\dot{\sigma}_{i}\left(U_{i}\right)=\left(1-\xi_{i}\right) k_{i} \dot{U}_{i} & \text { if the } i \text {-th point is plastic, }
\end{array}
$$

and $\sigma_{N+1}=0$. Definition of "elastic" and "plastic" is exactly the same as in the single system, except the case when both $\dot{U}_{i}$ and $\ddot{U}_{i}$ vanish at $t=t_{0}$ and we can not determine the state for $t \geq t_{0}$. In the single system, we do not have to bother about such problem since if it should happen, $u(t)=u\left(t_{0}\right), \sigma=0$ is the only possible solution for $t \geq t_{0}$. The situation is, however, almost the same in the multiple system too. In fact, we have the following theorem.

Theorem 2.1. Let $U_{i}, \sigma_{i}$ be the solution of the initial value problem for (2.1). Assume that at $t=t_{0}$ the points $\left(U_{i}(t), \sigma_{i}(t)\right)\left(i=i_{1}, \ldots, i_{r}\right)$ lie on one of the lines

$$
\sigma_{i}\left(U_{i}\right)=k_{i} U_{i}-\xi_{i} k_{i}\left(U_{i} \pm \bar{U}_{i}^{(0)}\right)
$$

in the $\left(U_{i}, \sigma_{i}\left(U_{i}\right)\right)$ plane respectively, where $\bar{U}_{i}^{(0)}(>0)$ denotes the yield strain of the i-th masspoint, and moreover that

$$
\stackrel{(l)}{U}_{i}\left(t_{0}+0\right)=0 \quad(1 \leq l \leq k) \quad(k \geq 2)
$$

for $i=i_{1}, \ldots, i_{r}$. Then $U_{i}^{(k+1)}\left(t_{0}+0\right)$ for such $i$ is determined independently of the form of $\dot{\sigma}_{i}\left(i=i_{1}, \ldots, i_{r}\right)$ for $t \geq t_{0}$, provided that the other $\dot{\sigma}_{i}$ 's are already decided for $t \geq t_{0}$.

Proof. Use an induction on $k$, considering the facts that $\sigma_{j}^{(k-1)}\left(t_{0}+0\right)$ $=$ constant $\cdot{ }^{(k-1)} U_{j}\left(t_{0}+0\right)=0$ if $j$ is included in $\left(i_{1}, \ldots, i_{r}\right)$ and

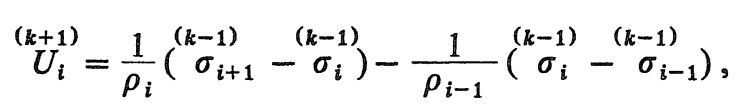

and that the sign of the non-vanishing lowest $\stackrel{(k)}{U}_{i}\left(t_{0}+0\right)$ can determine the state of the $i$-th point for $t \geq t_{0}$, since the point $\left(U_{i}(t), \sigma_{i}(t)\right)$ in the $\left(U_{i}, \sigma_{i}\left(U_{i}\right)\right)$ plane moves at $t=t_{0}$ to a definite direction determined by this sign.

Applying this theorem repeatedly, we can determine the state of each point for $t \geq t_{0}$, except a very special case. If $U_{i}^{(k)}$ should vanish for any $k$ at $t=t_{0}+0$, how should we determine the subsequent state? In this case we can define free for $t \geq t_{0}$ (we thus define that the point is plastic). The reason is this: The solu- 
tion $\left\{U_{i}\right\}$ is analytic until some point $j(\neq i)$ changes its state. Therefore, $\stackrel{(k)}{U_{i}}\left(t_{0}+0\right)=0$ (for any $k \geq 1$ ) implies that $U_{i}=$ constant until that time, which implies the solution does not depend on if we define the $i$-th point to be plastic or elastic.

Our problem is hence well posed and has a unique $C^{2}$-class solution for any $t>0$.

2.2. Energy of the multiple masspoint system. To derive an energy form for the multiple system, we define that the $i$-th masspoint is at $\operatorname{stage}\left(m_{i}\right)$ as the same way in the single system, replacing $u$ and $\sigma$ by $U_{i}$ and $\sigma_{i}\left(U_{i}\right)$. (Remark: There is a formal possibility that there exist infinitely many changes of the state in finite time interval. In this case the points $\left(U_{i}^{(j)}, \sigma_{i}\left(U_{i}^{(j)}\right)\right)(j=1,2, \ldots)$ have an accumulation point on the line defined (2.2), without making any hysteresis loop. For the following discussion, however, we can assume without loss of generality that the number of the state change is finite in finite time interval, since, if such accumulation should happen, we can skip all stages near the accumulation point in numbering the stage. Note that these stages give no influence on both the equation and the energy form.)

We say that the system is at $\operatorname{stage}(m)\left(m=\left(m_{1}, \ldots, m_{N}\right)\right)$, if the $i$-th masspoint is at stage $\left(m_{i}\right)$. Corresponding to Theorem 1.1 and Theorem 1.2, we have the following two theorems.

Theorem 2.2. At stage $(m)$, the equation of motion of the multiple masspoint system is represented as follows.

$$
\begin{gathered}
\rho_{i} \ddot{u}_{i}+\left[k_{i} U_{i}-\xi_{i} k_{i} \sum_{j=0}^{m_{i}}(-1)^{j}\left(U_{i}-U_{i}^{(j)}\right)\right] \\
-\left[k_{i+1} U_{i+1}-\xi_{i+1} k_{i+1} \sum_{j=0}^{m_{i+1}}(-1)^{j}\left(U_{i+1}-U_{i+1}^{(j)}\right)\right]=0, \\
(i=1, \ldots, N)
\end{gathered}
$$

where $U_{i}^{(j)}$ denotes the displacement of the $i$-th point when it enters in stage (j). Here we assume that $k_{N+1}=0$.

Theorem 2.3. Let $E_{m}(t)$ be defined $b \mathrm{y}$

$$
E_{m}(t)=\frac{1}{2} \sum_{1}^{N}\left[\rho_{i}\left(\dot{u}_{i}\right)^{2}+k_{i} U_{i}^{2}-\xi_{i} k_{i} \sum_{j=0}^{m_{i}}(-1)^{j}\left(U_{i}-U_{i}^{(j)}\right)^{2}\right] .
$$

Then the following equality holds at stage $(m)$.

$$
E_{m}(t)=E_{0}
$$


where $E_{0}$ is the initial energy.

The first theorem will be evident. Also, it is easy to see

$$
\begin{aligned}
\sum_{i} & {\left[\zeta_{i} k_{i} \sum_{j=0}^{m_{2}}(-1)^{j}\left(U_{i}-U_{i}^{(j)}\right)-\check{\zeta}_{i+1} k_{i+1} \sum_{j=0}^{m_{l}+1}(-1)^{j}\left(U_{i+1}-U_{i+1}^{(j)}\right)\right] \dot{u}_{i} } \\
= & -\frac{1}{2}\left[\sum_{i} \xi_{i} k_{i} \sum_{j=0}^{m_{l}}(-1)^{j}\left(U_{i}-U_{i}^{(j)}\right)^{2}\right]_{t} .
\end{aligned}
$$

The quantity $E_{i n}(t)$ is hence constant through all stages, so that it is equal to the initial energy. The second theorem is thus proved.

2.3. A weak form of the equation of motion. Hereafter we assume that $k_{i}$, $\zeta_{i}$ and $\bar{U}_{i}^{(0)}$ are independent of $i$ and denote them by $k, \zeta$ and $\bar{U}^{(0)}$, respectively. As in the single system, the present problem can be represented by a weak form including an inequality.

Let $v, \sigma$ and $\alpha$ be $N$-dimensional vector functions which are differentiable in $t \geq 0$. Let $K$ be a set of $N$-dimensional vector functions which are continuous and within $\sigma_{0}$-neighborhood of $\alpha$, that is,

$$
K=K_{\alpha}=\left\{\tau \in C(T)^{N} ; \quad \operatorname{Max}_{i}\left|\tau_{i}-\alpha_{i}\right| \leq \sigma_{0} \quad \text { for any } t \in T\right\} ; \sigma_{0}=k \bar{U}^{(0)} .
$$

Theorem 2.4. Introduce $v$ by $\dot{U}_{i}=v_{i}-v_{i-1}\left(v_{0} \equiv 0\right)$. The initial value problem of the multiple masspoint system is equivalent to the following problem: Seek $v, \sigma, \alpha$ which are differentiable and satisfy

$$
\left\{\begin{array}{l}
(\dot{\sigma}-k \dot{U}, \tau-\sigma)_{E^{N}} \geq 0 \quad \text { for any } \quad \tau \in K, \\
\dot{\alpha}=\left(1-\frac{1}{\xi}\right)(\dot{\sigma}-k \dot{U}) \\
\rho_{i} \dot{v}_{i}+\sigma_{i}-\sigma_{i+1}=0 \quad i=1, \ldots, N
\end{array}\right.
$$

and $\sigma \in K, \sigma(0)=0, \sigma_{N+1}=0, \alpha(0)=0, v(0)=\dot{u}(0)$.

Proof. Let $\left\{u_{i}\right\}$ be the solution of the previous problem. Put $v=\dot{u}$. At time $t$, let the points $\left(i_{1}, \ldots, i_{r}\right)$ and $\left(i_{r+1}, \ldots, i_{N}\right)$ be elastic and plastic, respectively, or equivalently,

$$
\begin{array}{ll}
\dot{\sigma}_{i}=k \dot{U}_{i} & \text { for } \quad i=i_{1}, \ldots, i_{r} \\
\dot{\sigma}_{i}=(1-\xi) k \dot{U}_{i} & \text { for } \quad i=i_{r+1}, \ldots, i_{N} .
\end{array}
$$

If point $i$ is elastic (resp. plastic), the equation for $\alpha_{i}$ is

$$
\dot{\alpha}_{i}=0 \quad\left(\text { resp. }=\dot{\sigma}_{i}\right),
$$

so that $\alpha$ is nothing but the parameter representing the center of the yield surface. Also, 


$$
(\dot{\sigma}-k \dot{U}, \tau-\sigma)=-\xi k \sum_{i=i_{r+1}}^{i_{N}}\left(\dot{U}_{i}, \tau_{i}-\sigma_{i}\right) .
$$

However, if point $i$ is plastic, we have

$$
\begin{aligned}
& \text { if } \dot{U}_{i}>0 \text {, then } \sigma_{i}=\alpha_{i}+\sigma_{0}, \\
& \text { if } \dot{U}_{i}<0 \text {, then } \sigma_{i}=\alpha_{i}-\sigma_{0},
\end{aligned}
$$

which imply

$$
\dot{U}_{i}\left(\tau_{i}-\sigma_{i}\right) \leq 0 \quad \text { for any } \quad \tau \in K,
$$

and hence,

$$
(\dot{\sigma}-k \dot{U}, \tau-\sigma) \geq 0 \quad \text { for any } \quad \tau \in K .
$$

Therefore $v=\dot{u}$ and $\sigma$ satisfy (2.4). Since the uniqueness of the solution of (2.4) can be proved by just the same way as in the single system, the proof is complete.

2.4. Energy inequality (1). To prove the existence of a solution for the continuous problem, Duvaut-Lions [1] have used a penalization technique, introducing an elasto-visco-plastic problem. In our problem, however, we can directly get the required estimates from this discrete problem. First, a basic estimate is obtained as follows.

Put $\tau=\alpha$ in the inequality of (2.4). Substituting the equation for $\alpha$, we get

$$
\begin{array}{r}
0 \leq \eta\left(\dot{\sigma}-k \dot{U}, \int_{0}^{t}[\dot{\sigma}-k \dot{U}] d t\right)-(\dot{\sigma}, \sigma)+k(\dot{U}, \sigma) \\
\left(\eta=1-\frac{1}{\xi}\right) .
\end{array}
$$

Since $(\dot{U}, \sigma)=\sum\left(v_{i}, \sigma_{i}-\sigma_{i+1}\right)=-\sum \rho_{i}\left(v_{i}, \dot{v}_{i}\right)=-1 / 2 \sum \rho_{i}\left(v_{i}\right)_{t}^{2}$, we have

$$
\begin{aligned}
& \left(-\frac{1}{2 \eta}\right)\|\alpha\|^{2}+\frac{1}{2}\|\sigma\|^{2}+\frac{k}{2} \sum_{i} \rho_{i}\left(v_{i}\right)^{2} \\
& \leq \frac{k}{2} \sum_{i} \rho_{i} v_{i}^{2}(0) .
\end{aligned}
$$

2.5. Energy inequality (2). We shall estimate some higher "derivatives". We differentiate the second equation in (2.4):

$$
\rho_{i} \ddot{v}_{i}+\dot{\sigma}_{i}-\dot{\sigma}_{i+1}=0 \text {. }
$$

Multiplying by $\dot{v}_{i}$ and summing on $i$ we have, taking into account Theorem 2.2,

$$
\sum_{i} \frac{\rho_{i}}{2}\left(\dot{v}_{i}\right)_{t}^{2}+\frac{k}{2} \sum_{i}\left(1-\xi \sum_{j=0}^{m_{i}}(-1)^{j}\right)\left(\dot{U}_{i}^{2}\right)_{t}=0
$$


We have to be careful in integrating this equation. Assume that the system is now at $\operatorname{stage}(m)$ and this stage began at $t=t_{0}$. Let us also assume that the points

$$
\begin{aligned}
& i=i_{1}, \ldots, i_{p} \quad \text { change from elastic to plastic, } \\
& i=i_{p+1}, \ldots, i_{q} \quad \text { change from plastic to elastic }
\end{aligned}
$$

at this moment and other points remain unchanged. If the preceding stage is stage $\left(m^{\prime}\right)$, we see

$$
\begin{aligned}
m_{i} & =m_{i}^{\prime}, \quad i \neq i_{1}, \ldots, i_{q}, \\
& =m_{i}^{\prime}+1, \quad i=i_{1}, \ldots, i_{q} .
\end{aligned}
$$

Define

$$
\bar{E}_{m}(t)=\sum_{i}\left[\frac{\rho_{i}}{2}\left(\dot{v}_{i}\right)^{2}+\frac{k}{2}\left(1-\xi \sum_{j=0}^{m_{i}}(-1)^{j}\right) \dot{U}_{i}^{2}\right] .
$$

Then for any $t^{\prime}$ and $t$ near $t_{0}\left(t^{\prime}<t_{0}<t\right)$, we have

$$
\begin{aligned}
\bar{E}_{m}(t) & -\bar{E}_{m^{\prime}}\left(t^{\prime}\right)=\bar{E}_{m}\left(t_{0}\right)-\bar{E}_{m^{\prime}}\left(t_{0}\right) \\
= & \sum_{i}\left[\frac{\rho_{i}}{2}\left(\dot{v}_{i}\right)^{2}\left(t_{0}\right)+\frac{k}{2}\left(1-\xi \sum_{j=0}^{m_{i}}(-1)^{j}\right) \dot{U}_{i}^{2}\left(t_{0}\right)\right] \\
& -\sum_{i}\left[\frac{\rho_{i}}{2}\left(\dot{v}_{i}\right)^{2}\left(t_{0}\right)+\frac{k}{2}\left(1-\xi \sum_{j=0}^{m_{i}^{\prime}}(-1)^{j}\right) \dot{U}_{i}^{2}\left(t_{0}\right)\right] .
\end{aligned}
$$

Since $\dot{v}_{i}$ is continuous at $t=t_{0}$, the terms on this value cancelled. Also, the terms on $\dot{U}_{i}^{2}\left(t_{0}\right)$ for $i \neq i_{1}, \ldots, i_{p}$ are cancelled. Therefore, the above quantity is the sum of

$$
\begin{gathered}
\frac{k}{2}\left(1-\xi \sum_{j=0}^{m_{i}}(-1)^{j}\right) \dot{U}_{i}^{2}\left(t_{0}\right)-\frac{k}{2}\left(1-\xi \sum_{j=0}^{m_{i}^{\prime}}(-1)^{j}\right) \dot{U}_{i}^{2}\left(t_{0}\right) \\
=-\frac{k \xi}{2} \dot{U}_{i}^{2}\left(t_{0}\right) \leq 0 \quad\left(m_{i} \text { : even }\right)
\end{gathered}
$$

for $i=i_{1}, \ldots, i_{p}$, which implies $\bar{E}_{m}(t) \leq \bar{E}_{m^{\prime}}\left(t^{\prime}\right)$ and hence

$$
\sum_{i}\left[\frac{\rho_{i}}{2}\left(\dot{v}_{i}\right)^{2}+\frac{k}{2}(1-\xi) \dot{U}_{i}^{2}\right](t) \leq \sum_{i}\left[\frac{\rho_{i}}{2}\left(\dot{v}_{i}\right)^{2}+\frac{k}{2} \dot{U}_{i}^{2}\right](0) \text {. }
$$

Once this estimate is obtained, other derivatives are easily estimated. The result is as follows. Let $\bar{E}_{0}$ be the quantity of the right side of (2.5). Then we have

$$
\left\{\begin{array}{l}
\sum_{i} \dot{\sigma}_{i}^{2} \leq 2 k \bar{E}_{0}, \\
\sum_{i}\left(\sigma_{i+1}-\sigma_{i}\right)^{2} \leq 2 \operatorname{Max} \rho_{i} \bar{E}_{0}, \\
\sum_{i} \dot{\alpha}_{i}^{2} \leq \frac{8(1-\xi) k}{\xi^{2}} \bar{E}_{0} .
\end{array}\right.
$$




\section{§3. Elastic-Plastic Vibration of a Rod}

3.1. A weak form of the equation of motion. We introduce a weak form of the original problem and show that this form is a natural extension of the equation of motion of the multiple masspoint system considered in the previous sections. Let $\Omega=(0,1)$ and define

$$
K=K_{\alpha}=\left\{\tau \in L^{\infty}\left(T ; L_{2}(\Omega)\right) ; \text { a.e. } T,|\tau-\alpha| \leq \sigma_{0} \quad \text { a.e. } \quad \Omega\right\}
$$

for $\alpha \in L^{\alpha}\left(T ; L_{2}(\Omega)\right)$. Then our problem is:

Find $(v, \sigma, \alpha)$ such that

$$
v, \sigma \in L^{\infty}\left(T ; W_{2}^{1}(\Omega)\right), \quad \dot{v}, \dot{\sigma}, \alpha, \dot{\alpha} \in L^{\infty}\left(T ; L_{2}(\Omega)\right)
$$

and a.e. $T$,

$$
\left\{\begin{array}{l}
\left(\dot{\sigma}-k v_{x}, \tau-\sigma\right)_{L_{2}(\Omega)} \geq 0 \quad \text { for any } \quad \tau \in K, \\
\dot{\alpha}=\left(1-\frac{1}{\xi}\right)\left(\dot{\sigma}-k v_{x}\right) \\
\dot{v}-\sigma_{x}=0,
\end{array}\right.
$$

where $\sigma \in K, v(0, x)=a(x)$ : given, $v(t, 0)=0, \sigma(0, x)=0, \sigma(t, 1)=0, \alpha(0, x)=0$.

It is evident that if the original problem has a classical solution, then $v=\dot{u}$ and $\sigma$ satisfy this equation, $\alpha$ being the parameter representing the center of yield surface (two points, in this case too).

3.2. Finite element approximations. We use the finite element solutions and pass to the limit to get the solution for (3.1).

We first divide the interval $\Omega$ into $N$ elements of equal length $h$. Let $i$ be the point with coordinate $i h(i=0,1, \ldots, N)$ and $e_{i}$ the element $[(i-1) h, i h]$, We use three basis functions:

$$
\hat{\varphi}_{i}(x) \text { : piecewise linear basis, }
$$

$\therefore \bar{\varphi}_{i}(x):$ characteristic function of $\left[i h-\frac{h}{2}, i h+\frac{h}{2}\right]$,

$\overline{\bar{\varphi}}_{i}(x)$ : characteristic function of element $e_{i}$.

Then the simplest finite element approximation to the original problem is

$$
\left\{\begin{array}{l}
\left(\ddot{\bar{u}}, \bar{\varphi}_{i}\right)+\sum_{e}\left(\sigma(\hat{u}), \hat{\varphi}_{i, x}\right)_{e}=0 \quad i=1, \ldots, N \\
\dot{\sigma}(\hat{u})= \begin{cases}k \dot{\hat{u}}_{x} & \text { in elastic region } \\
(1-\xi) k \dot{\hat{u}}_{x} & \text { in plastic region, }\end{cases}
\end{array}\right.
$$


where

$$
\begin{aligned}
& \bar{u}=\sum_{i=1}^{N} u_{i}(t) \bar{\varphi}_{i}(x) \\
& \hat{\imath}=\sum_{i=1}^{N} u_{i}(t) \hat{\varphi}_{i}(x),
\end{aligned}
$$

and $u_{i}(0)=0, \dot{u}_{i}(0)=a(i h) . \quad(a(x)$ is assumed to be smooth and $a(0)=0$.)

Since $\sigma$ is constant on each element, we put

$$
\sigma_{i}=\left.\sigma(\hat{u})\right|_{e_{i}},
$$

to get

$$
\sum_{e}\left(\sigma(\hat{u}), \hat{\varphi}_{i, x}\right)_{e}=\sigma_{i}-\sigma_{i+1} \quad\left(\sigma_{N+1}=0\right) .
$$

Then the finite element equation (3.2) is written as

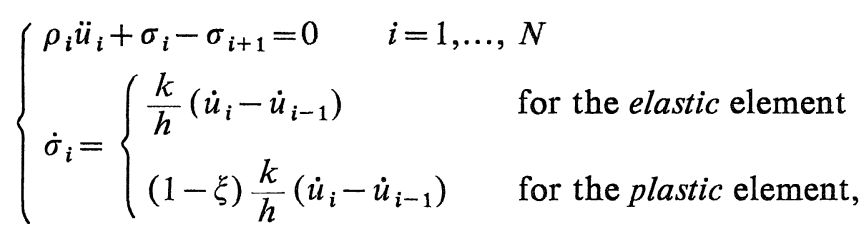

where $\rho_{i}=h$ for $i \neq N$ and $=h / 2$ for $i=N, u_{0}=0$, which is just the same equation considered in the previous sections.

3.3. Convergence of the finite element solutions. We shall show that a limit of the finite element solutions is a solution of (3.1). Define

$$
\overline{\bar{\sigma}}=\sum_{i=1}^{N} \sigma_{i} \varphi_{i}, \quad \hat{\sigma}_{*}=\sum_{i=0}^{N} \sigma_{i+1} \hat{\varphi}_{i} .
$$

Then the energy inequalities for the multiple system imply

$$
\left.\begin{array}{l}
\overline{\bar{\sigma}}, \dot{\overline{\bar{\sigma}}},\left(\hat{\sigma}_{*}\right)_{x} \\
\bar{v}, \dot{\bar{v}}, \hat{v}_{x}(v=\dot{u}) \\
\bar{\alpha}, \overline{\bar{\alpha}}
\end{array}\right\} \text { remain in a bounded set of } L^{\infty}\left(T ; L_{2}(\Omega)\right),
$$

as $h \rightarrow 0$. Therefore we can select a sequence such that

$$
\begin{array}{ll}
\dot{\bar{\sigma}},\left(\hat{\sigma}_{*}\right)_{x} \rightarrow \dot{\sigma}, \sigma_{x} & \text { weakly* in } L^{\infty}\left(T ; L_{2}(\Omega)\right), \\
\overline{\bar{\sigma}} \rightarrow \sigma & \text { strongly in } L_{2}(T \times \Omega),
\end{array}
$$

and $\sigma(t, 1)=0, \sigma(0, x)=0$,

$$
\begin{array}{ll}
\dot{\bar{v}}, \hat{v}_{x} \rightarrow \dot{v}, v_{x} & \text { weakly* in } L^{\infty}\left(T ; L_{2}(\Omega)\right), \\
\bar{v} \rightarrow v & \text { strongly in } L_{2}(T \times \Omega),
\end{array}
$$


and $v(t, 0)=0, v(0, x)=a(x)$, and

$$
\bar{\alpha}, \dot{\bar{\alpha}} \rightarrow \alpha, \dot{\alpha} \quad \text { weakly* in } L^{\infty}\left(T ; L_{2}(\Omega)\right) .
$$

(We used some elementary properties of the finite elements.) Let $t$ be an arbitrary point in $[0, T]$. If $\beta$ and $\dot{\beta}$ are included in $L^{\infty}\left(T ; L_{2}(\Omega)\right)$ with $\beta(0)=0$, then $\beta(t)$ is well defined as a function of $L_{2}(\Omega)$ and holds

$$
\|\beta(t)\|_{L_{2}(\Omega)} \leq C\|\dot{\beta}\|_{L_{2}(T \times \Omega)},
$$

where $C$ is a constant independent of $\beta$. Therefore, we can assume that

$$
\bar{\alpha}(T) \rightarrow \alpha(T) \quad \text { weakly in } L_{2}(\Omega),
$$

and also $\alpha(0)=0$. According to Theorem 2.4, we can rewrite the system (3.3) as follows.

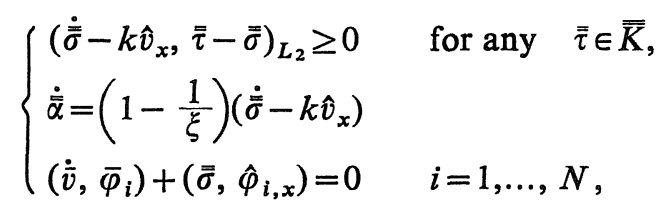

where

$$
\overline{\bar{K}}=\left\{\overline{\bar{\tau}} ; \operatorname{Max}_{\Omega}|\overline{\bar{\tau}}-\overline{\bar{\alpha}}| \leq \sigma_{0} \quad \text { for any } \quad t \in T\right\} .
$$

Let $\varphi$ be an arbitrary function of $C^{\infty}(T \times \Omega)$ with support in $T \times \Omega$. Let $\bar{\varphi}$ and $\hat{\varphi}$ be the interpolating functions:

$$
\begin{aligned}
& \bar{\varphi}=\sum_{i} \varphi(t: i h) \bar{\varphi}_{i} \\
& \hat{\varphi}=\sum_{i} \varphi(t: i h) \hat{\varphi}_{i} .
\end{aligned}
$$

As well known, $\|\varphi-\bar{\varphi}\|_{L_{2}(T \times \Omega)} \rightarrow 0,\|\varphi-\hat{\varphi}\|_{W_{2}^{1}(T \times \Omega)} \rightarrow 0$ as $h \rightarrow 0$. Therefore we have

$$
\begin{aligned}
\int_{0}^{T}\left(\dot{v}-\sigma_{x}, \varphi\right) d t=\int_{0}^{T}(\dot{v}-\dot{\bar{v}}, \varphi) d t+\int_{0}^{T}(\dot{v}-\dot{\bar{v}}, \bar{\varphi}-\varphi) d t \\
+\int_{0}^{T}\left(\sigma-\overline{\bar{\sigma}}, \hat{\varphi}_{x}\right) d t+\varepsilon_{h} \quad\left(\varepsilon_{h} \rightarrow 0 \text { as } h \rightarrow 0\right),
\end{aligned}
$$

from which we can conclude that

$$
\dot{v}-\sigma_{x}=0 \quad \text { a.e. } T .
$$

By the same way we have

$$
\dot{\alpha}=\left(1-\frac{1}{\xi}\right)\left(\dot{\sigma}-k v_{x}\right) \quad \text { a.e. } T .
$$


To prove the inequality, take an arbitrary $\tau \in K$. Then $\tau$ is written generally as

$$
\tau=\alpha+\sigma_{0} \theta
$$

where $\theta \in L^{\infty}\left(T ; L_{2}(\Omega)\right)$ and satisfies a.e. $T$,

$$
|\theta| \leq 1 \quad \text { a.e. } \Omega \text {. }
$$

For this $\theta$ we can find a function $\bar{\theta}$ on $T \times \Omega$ which is constructed by the system of functions $\left\{\overline{\bar{\varphi}}_{i}\right\}$ such that for given $\varepsilon$,

$$
\|\theta-\bar{\theta}\|_{L_{2}(T \times \Omega)}<\varepsilon, \quad|\bar{\theta}| \leq 1 \quad \text { in } \quad T \times \Omega .
$$

For example, take Friedrichs' mollifier $\rho_{\delta}$ and make the convolution

$$
\theta_{\delta}=\rho_{\delta} * \theta \text {. }
$$

Then clearly $\left|\theta_{\delta}\right| \leq 1$ in $T \times \Omega$, and

$$
\left\|\theta-\theta_{\delta}\right\|_{L_{2}(T \times \Omega)}<\frac{\varepsilon}{2} \quad \text { for sufficiently small } \delta \text {. }
$$

Let $\bar{\theta}$ be the interpolation of $\theta_{\delta}$ by the coordinate functions $\left\{\bar{\varphi}_{i}\right\}$, then clearly $|\bar{\theta}| \leq 1$ and for sufficiently small $h$

$$
\left\|\theta_{\delta}-\overline{\bar{\theta}}\right\|_{L_{2}(T \times \Omega)}<\frac{\varepsilon}{2} .
$$

This $\bar{\theta}$ is hence the desired function.

Now in the inequality of (3.4) put

$$
\overline{\bar{\tau}}=\overline{\bar{\alpha}}+\delta_{0} \overline{\bar{\theta}}
$$

(if necessary, take a subsequence) and integrate on $t$. We then have

$$
0 \geq \frac{1}{2}\|\overline{\bar{\alpha}}\|^{2}(T)+\sigma_{0} \int_{0}^{T}(\dot{\bar{\alpha}}, \overline{\bar{\theta}}) d t-\int_{0}^{T}(\dot{\bar{\alpha}}, \overline{\bar{\sigma}}) d t .
$$

When $h \rightarrow 0$ we have

$$
\begin{aligned}
& \underline{\lim }\|\overline{\bar{\alpha}}\|^{2}(T) \geq\|\alpha\|^{2}(T), \\
& \int_{0}^{T}(\dot{\overline{\bar{\alpha}}}, \overline{\bar{\theta}}) d t=\int_{0}^{T}(\dot{\overline{\bar{\alpha}}}, \theta) d t+\int_{0}^{T}(\dot{\overline{\bar{\alpha}}}, \bar{\theta}-\theta) d t \rightarrow \int_{0}^{T}(\dot{\alpha}, \theta) d t, \\
& \int_{0}^{T}(\dot{\overline{\bar{\alpha}}}, \overline{\bar{\sigma}}) d t=\int_{0}^{T}(\dot{\overline{\bar{\alpha}}}, \sigma) d t+\int_{0}^{T}(\dot{\overline{\bar{\alpha}}}, \overline{\bar{\sigma}}-\sigma) d t \rightarrow \int_{0}^{T}(\dot{\alpha}, \sigma) d t .
\end{aligned}
$$

Therefore (3.5) implies 


$$
\begin{aligned}
0 & \geq \frac{1}{2}\|\alpha\|^{2}(T)+\sigma_{0} \int_{0}^{T}(\dot{\alpha}, \theta) d t-\int_{0}^{T}(\dot{\alpha}, \sigma) d t \\
& =\int_{0}^{T}(\dot{\alpha}, \tau-\sigma) d t,
\end{aligned}
$$

for arbitrary $\tau \in K$. Take $t \in(0, T)$ and for arbitrary $\bar{\tau} \in K$ define

$$
\tau=\left\{\begin{array}{lll}
\bar{\tau} & \text { in } T_{\varepsilon} \\
\sigma & \text { in } T-T_{\varepsilon} \quad(\varepsilon>0),
\end{array}\right.
$$

where $T_{\varepsilon}=[t-\varepsilon / 2, t+\varepsilon / 2]$. Then clearly $\tau \in K$ and

$$
0 \geq \frac{1}{\varepsilon} \int_{0}^{T}(\dot{\alpha}, \tau-\sigma) d t=\frac{1}{\varepsilon} \int_{T_{\varepsilon}}(\dot{\alpha}, \bar{\tau}-\sigma) d t .
$$

Therefore letting $\varepsilon \rightarrow 0$ we have

$$
(\dot{\alpha}, \bar{\tau}-\sigma) \leq 0 \quad \text { a.e. } T,
$$

for arbitrary $\bar{\tau} \in K$. Since $\sigma \in K$, the existence of a solution of (3.1) is proved. Uniqueness of the solution of this problem is proved by just the same way for the single system. Hence the whole sequence $(\bar{v}, \bar{\sigma}, \bar{\alpha})$ converges to the exact solution and we finally have

Theorem 3.1. There exists a unique solution $(v, \sigma, \alpha)$ for (3.1), which is the limit of the finite element solutions and, at the same time, regarded as the limit of the solutions for the multiple masspoint systems.

\section{References}

[1] Duvaut, G. and Lions, J. L., Inequalities in Mechanics and Physics, Springer, 1976.

[2] Kachanov, L. M., Foundations of the Theory of Plasticity, North-Holland, 1971.

[3] Johnson, C., On plasticity with hardening, J. Math. Anal. Appl., 62 (1978), 325-336. 